\title{
Paranoia and conspiracy: group cohesion increases harmful intent attribution in the Trust Game
}

\author{
Anna Greenburgh ${ }^{1}$, Vaughan Bell ${ }^{2,3}$, Nichola Raihani ${ }^{\text {Corresp. } 1}$ \\ 1 Department of Experimental Psychology, University College London, London, United Kingdom \\ 2 Department of Clinical, Education and Health Psychology, University College London, London, United Kingdom \\ 3 Psychological Interventions Clinic for Outpatients with Psychosis (PICuP), South London \& Maudsley NHS Foundation Trust, London, United Kingdon \\ Corresponding Author: Nichola Raihani \\ Email address: n.raihani@ucl.ac.uk
}

Current theories argue that hyper-sensitisation of social threat perception is central to paranoia. Affected people often also report misperceptions of group cohesion (conspiracy) but little is known about the cognitive mechanisms underpinning this conspiracy thinking in live interactions. In a pre-registered experimental study, we used a large-scale game theory approach ( $\mathrm{N}>1000)$ to test whether the social cohesion of an opposing group affects paranoid attributions in a mixed online and lab-based sample. Participants spanning the full population distribution of paranoia played as proposers in a modified Trust Game: they were allocated a bonus and chose how much money to send to a pair of responders which was quadrupled before reaching these responders. Responders decided how much to return to the proposers through the same process. Participants played in one of two conditions: against a cohesive group who communicated and arrived at a joint decision, or a non-cohesive group who made independent decisions. After the exchange, proposers rated the extent to which the responders' decisions were driven by (i) selfinterest and (ii) intent to harm. Although the true motives are ambiguous, cohesive responders were reliably rated by participants as being more strongly motivated by intent to harm, indicating that group cohesion affects social threat perception. Highly paranoid participants attributed harmful intent more strongly overall but were equally reactive to social cohesion as other participants. This suggests that paranoia involves a generally lowered threshold for social threat detection but with an intact sensitivity for cohesionrelated group characteristics. 
1 Title: Paranoia and conspiracy: group cohesion increases harmful intent attribution in the

\section{Trust Game}

3

4 Anna Greenburgh*1, Vaughan Bell ${ }^{2,3}$, and Nichola Raihani ${ }^{1}$

5

$6{ }^{1}$ Department of Experimental Psychology, University College London, London, United Kingdom

72 Department of Clinical, Education and Health Psychology, University College London,

8 London, United Kingdom

$9{ }^{3}$ Psychological Interventions Clinic for Outpatients with Psychosis (PICuP), South London and

10 Maudsley NHS Foundation Trust, London, United Kingdom

11

12 *Corresponding author information: Nichola Raihani,n.raihani@ucl.ac.uk 


\section{Abstract}

16 Current theories argue that hyper-sensitisation of social threat perception is central to paranoia.

17 Affected people often also report misperceptions of group cohesion (conspiracy) but little is

18 known about the cognitive mechanisms underpinning this conspiracy thinking in live

19 interactions. In a pre-registered experimental study, we used a large-scale game theory approach

$20(\mathrm{~N}>1000)$ to test whether the social cohesion of an opposing group affects paranoid attributions

21 in a mixed online and lab-based sample. Participants spanning the full population distribution of

22 paranoia played as proposers in a modified Trust Game: they were allocated a bonus and chose

23 how much money to send to a pair of responders which was quadrupled before reaching these

24 responders. Responders decided how much to return to the proposers through the same process.

25 Participants played in one of two conditions: against a cohesive group who communicated and

26 arrived at a joint decision, or a non-cohesive group who made independent decisions. After the

27 exchange, proposers rated the extent to which the responders' decisions were driven by (i) self-

28 interest and (ii) intent to harm. Although the true motives are ambiguous, cohesive responders

29 were reliably rated by participants as being more strongly motivated by intent to harm, indicating

30 that group cohesion affects social threat perception. Highly paranoid participants attributed

31 harmful intent more strongly overall but were equally reactive to social cohesion as other

32 participants. This suggests that paranoia involves a generally lowered threshold for social threat

33 detection but with an intact sensitivity for cohesion-related group characteristics. 
Introduction

37

38 Although paranoia is the most common positive symptom of psychosis (Freeman et al, 2007), it 39 is also present to varying degrees in the general population. Paranoia can range from mildly 40 exaggerated concerns about how others see us, to frank and disabling paranoid delusions of 41 conspiratorial harm (Freeman and Garety, 2014; Taylor et al, 2016; Elahi et al 2017).

42 _. Estimations suggest a third of the general population frequently experience paranoid 43 thoughts (Freeman et al, 2005). Paranoid ideation follows an exponential distribution where 44 increasingly severe thoughts are increasingly rare (Bebbington et al, 2013).

One well-established component of paranoia is an alteration to social threat perception. Patients 47 with persecutory delusions have better memory for threat-related words (Bentall et al, 1995) and actively paranoid patients diagnosed with schizophrenia over-perceive anger in neutral faces (Pinkham et al, 2011). Highly paranoid members of the general population over-estimate chances 50 of future victimisation (Jack and Egan, 2016) and are more likely to perceive harmful intent in 51 ambiguous social exchanges (Raihani and Bell, 2017).

52

53 Paranoia also frequently involves problems with accurately judging the intentions of groups, 54 rather than individuals (Raihani and Bell, 2019). In clinical studies, concerns about conspiracy 55 are a well-established component of paranoia that have been documented from early in the 56 history of psychiatry (Harper, 1994) and form part of current definitions (Oyebode, 2008). Here, 57 conspiracy perception refers to concerns about being persecuted by a group of others who are 
58 coordinated in attempts to harm the individual, but who do not correspond to any collection of

59 people with these aims - something Cameron (1959) conceptualised as the "paranoid

60 pseudocommunity'. This is distinct from belief in public conspiracy theories more broadly,

61 which describe conspiratorial explanations of important historical events that are not centred on

62 the believer (Douglas et al, 2017). Indeed, recent cross-cultural research highlights that that

63 while paranoia and conspiracy thinking (belief in public conspiracy theories) are associated, they

64 are divergent constructs (Imhoff and Lamberty, 2018).

65

66 Rather than viewing paranoia only as a symptom of a mental disorder, recent research has

67 suggested that it might be more accurate to view paranoia across the full spectrum of severity as

68 part of a normally-functioning human psychology that evolved in the context of detecting and

69 avoiding social threat (Green and Phillips, 2004; Raihani and Bell, 2019). Studies that have

70 examined social threat processing as a broader psychological mechanism suggest it is likely to be

71 supported by a specialised, evolved mechanism that is attuned to threats from both individuals

72 and groups. The Coalitional Index Model (Boyer et al, 2015) proposes that individuals can judge

73 cues of social threat and support and can integrate them to encode their own vulnerability in any

74 given environment. Under this hypothesis, activation of the 'coalitional safety index' triggers a

75 set of physiological, cognitive and behavioural responses attuned to the perceived degree of

76 social threat. Threat inputs to this mechanism include coalitional identity, as supported by

77 evidence that out-group cues increase anxiety and fear responses (e.g. Navarrete et al, 2009; Hart 78 et al, 2000). 
80 Such a psychological mechanism to detect social threat should be sensitive to the cohesiveness of

81 rival groups. More cohesive groups are more able to act towards a common goal. Therefore,

82 cohesive opponent groups should be perceived as more threatening than similar-sized but non-

83 cohesive groups (Boyer et al, 2015). Indeed, group entitativity (its perceived unity) increases

84 negative cognitive and behavioural responses towards the group (Campbell 1958; Hamilton and

85 Sherman, 1996). High-entitativity groups are also perceived as more morally suspicious

86 (Newheiser et al, 2012), conspiratorial (Grzesiak-Feldman and Suszek, 2008), more capable of

87 negative retaliation (Dasgupta, Banaji and Abelson, 1999) and evoke increased negative

88 stereotyping (Spencer-Rodgers, Hamilton and Sherman, 2007).

89

90 One clear implication of these studies is that individuals will exhibit increased threat responses

91 when in the presence of cohesive compared to non-cohesive groups. However, despite the clear

92 overlap with paranoia both conceptually and clinically, the effects of group cohesiveness on

93 threat perception have never been tested in this domain. Research is needed in this area to further

94 our understanding of social experience of paranoia, and how mechanisms of social cognition

95 function in paranoia.

96

97 Various hypotheses regarding paranoia and group cohesion emerge. The first is that paranoia

98 may involve an exaggerated reaction to group cohesion (a social over-sensitivity bias) so that

99 highly paranoid people will increase their attributions of harmful intent to cohesive compared to 100 non-cohesive groups, over and above the degree to which low-paranoia individuals do. Another

101 possible pattern is that, in addition to a general tendency to over-attribute harmful intent,

102 paranoia may involve a misperception of group cohesiveness such that all groups are perceived 
103 to be cohesive, suggesting that highly paranoid people will attribute equally-high levels of

104 harmful intent to cohesive and non-cohesive groups alike, unlike low-paranoia individuals who

105 will attribute greater levels of harmful intent to cohesive compared to non-cohesive groups.

106 Finally, it could be that paranoia simply involves a higher baseline of harmful intent attribution -

107 a general tendency to over-attribute harmful intent in social situations when compared to low-

108 paranoia individuals - but show the same pattern of reactivity to group cohesiveness as low109 paranoia individuals.

111 Here, we report a pre-registered study testing these predictions. We recruited a dual online and

112 offline sample, including a large online sample covering the population distribution of paranoid

113 ideation, and a lab-based panels of participants who formed cohesive and non-cohesive pairs to

114 respond to decisions made by the online participants. Previous studies have demonstrated that

115 large-scale game theory approaches are effective in capturing live paranoid attributions and 116 testing how paranoid attributions are modified by experimentally-induced social threat (Raihani 117 and Bell, 2017; Saalfeld et al, 2018). For example, modified Dictator Games have revealed that

118 individuals spanning the full paranoia spectrum rate unfair Dictators as intending more harm

119 than fair Dictators, signifying that fairness is used as a threat cue irrespective of an individual's

120 level of paranoid ideation (Raihani and Bell, 2017). Similarly, interacting with higher status, or 121 out-group partners also triggers exaggerated attributions of harmful intent in otherwise 122 ambiguous interactions (Saalfeld et al, 2018).

123

124 In the current study, participants played as proposers in an adapted Trust Game in one of two 125 conditions, where they interacted (i) against a pair of cohesive responders or (ii) against a same- 
126 sized group of non-cohesive responders. Participants were given an initial sum of money $(\$ 0.50)$

127 and were asked to indicate what proportion they would like to send $(\$ 0.25$ or $\$ 0.05)$ to a pair of

128 responders. Participants kept any of the money they did not send to the responders. Participants

129 were told that any amount they sent would be quadrupled by the experimenter, and that the

130 responders could decide whether to be fair and return half of the increased amount to the

131 participant or to be unfair and return nothing to the participant. Participants were randomly

132 allocated to either fair or unfair responder pairs.

133

134 The cohesiveness of the responder pair was characterised by whether responders could

135 communicate with one another or not. In the cohesive condition, responders had to agree on their

136 decision, whereas in the non-cohesive condition responders came to their decision

137 independently.

139 Participants chose how much to send to the responders and were then presented with the

140 responders' decision. After finding out how much the responders returned to them, participants

141 rated the extent to which they believed the responders' decisions to be driven by (i) self-interest

142 and (ii) harmful intent, using two separate slider scales. The true motives underpinning responder

143 decisions in this game are ambiguous and could plausibly reflect either self-interest (desire to

144 keep more money) or harmful intent (desire to exploit the participant). Previous work indicates

145 that paranoia positively predicts harmful intent attributions but not self-interest attributions in

146 similarly ambiguous settings (Raihani and Bell., 2017; Saalfeld et al, 2018). 


\section{Materials and Methods}

150 This study was approved by the UCL Ethics board, under project 3720/001. Participation was

151 voluntary and informed consent was obtained from all participants prior to their participation.

152

1532.1 Participants

154 The behavioural data were collected in August 2017; the GPTS data were collected in December 155 2016. We recruited 1,172 participants to the study (682 female). 1,164 of these participants were 156 recruited via Amazon Mechanical Turk (MTurk; http://www.mturk.com), whose decisions were 157 the focus of the analysis. Participants for this study were recruited from an existing database of 1583,500 participants for whom we already had data on pre-existing paranoia. This data was collected 159 in December 2016 for a previous study published in June 2017 (Raihani \& Bell, 2017). The 160 stability of paranoia scores over time was confirmed by re-collecting the GPTS scores of 420 161 participants from December 2016 in August $2017\left(r_{\mathrm{s}}=0.68, p<.001\right)$.

162

163 We aimed to recruit a minimum of 2,000 participants from this original database for this study

164 but we pre-registered a stopping rule that indicated we would stop data collection after responses 165 were slower than 20 per day. In addition, 8 participants took part in the study in the laboratory 166 and acted as paired responders to the online participants and were configured as cohesive 167 (communicated and made decisions together) or non-cohesive (did not communicate and made 168 decisions separately) pairs in deciding the response. All MTurk participants were based in the 169 US. The mean age of the (online) participants was $38.2(S D=12.3)$.

170

171 2.2 Design 
172 We used a between-subject design, where the independent variables were the cohesiveness of the

173 responder group in the Trust Game (non-cohesive / cohesive), fairness of responder decision (fair

174 / unfair) and paranoia (as measured by the GPTS). Dependent variables were attributions of i)

175 harmful intent and ii) self-interest made by proposers to the responder group. Predictions were

176 pre-registered at https://aspredicted.org/see_one.php?a id=4809.

177

178

\subsection{Materials and Procedure}

179 We used the Green et al, Paranoid Thought Scales (GPTS (Green et al, 2008)) to measure pre180 existing paranoid ideation. The GPTS is a reliable and valid scale for measuring paranoid 181 ideation across the full clinical and non-clinical spectrum. It is a 32-item scale, consisting of two 182 16-item subscales that measure feelings of social reference and persecution, respectively. 183 Participants were asked to endorse each item on a five-point Likert scale from 'Not at all' to

184 'Totally'. Responses are summed to provide final scores, which can range from 32-160, where 185 higher scores indicate a greater degree of paranoia. This variable will be referred to as 'pre186 existing paranoia'.

187

188 We used a modified Trust Game as the main behavioural paradigm. A standard Trust Game 189 (Berg et al, 1995) involves two participants, a proposer and a responder. The proposer is 190 allocated a sum of money and must decide how much to send to the responder. Any amount they 191 send is multiplied by a given factor, and the responder must decide how much of the resultant 192 amount to send back to the proposer. Our paradigm modified this design in two main fashions: 193 two responders played against a single proposer in each game, and we systemically manipulated 194 the cohesiveness of the responder pair. 
196 For the current study, proposers were recruited online and responders were lab-based

197 participants. On the online platform, proposers were allocated an initial sum of money $(\$ 0.50)$

198 and were asked to indicate what proportion this they would like to send $(\$ 0.25$ or $\$ 0.05)$ to the

199 pair of responders, where the remainder would be theirs to keep. Proposers were told that any

200 amount they sent to responders would be quadrupled, who decide how much of that amount to

201 return to the proposer. Responder decisions were pre-collected (proposers were aware of this),

202 and ex-post matching was used to assign proposers to responder pairs (Raihani, et al, 2013).

203 Responders were shown all possible decisions of proposers and were asked to decide how much

204 money they would return given each proposer decision.

205

206 Cohesiveness of the responder pair was characterised by whether they had to communicate and

207 make their decision as a pair. In the cohesive condition, responders communicated through an

208 online chat function and were required to come to a joint decision regarding much money to

209 return to the proposer. In the non-cohesive condition, responders did not communicate: they

210 came to their decision independently and they were subsequently matched with other responders

211 who made the same decision. The cohesiveness of responders was clearly communicated to the

212 proposers. This operationalization of cohesiveness was taken from key characteristics discussed

213 in the group cohesiveness and entitativity literature. This literature outlines many cues of group

214 cohesion, including group member interaction, agreement, and common goals (Lott and Lott,

215 1961; Campbell, 1958; Lickel et al, 2001). The online, anonymous nature of our design meant

216 that we were unable to use many cues that are associated with group cohesion, such as dress and

217 distinct language (Boyer et al, 2015). Despite using a subset of possible indicators of group 
218 cohesiveness, we confirmed that our manipulation impacted perception of cohesiveness through 219 a pre-registered manipulation check, which showed that 486 / 587 (83\%) participants in the 220 cohesive condition correctly perceived the responder pair as cohesive. For the non-cohesive 221 condition, 504 /564 (89\%) participants correctly perceived the responder pair as non-cohesive. 222 Binomial tests confirmed that participants reliably perceived the condition to which they were 223 assigned $(p<.001)$. Furthermore, correct perception of group cohesiveness did not vary with 224 paranoia.

225

226

After reading the instructions, participants were asked to answer three comprehension questions 227 to confirm they had understood the game (see Supplementary Materials). We conducted pre228 registered analyses to determine any effects of comprehension on the results (see below).

229

After making their decisions, proposers were informed of the responders' decision (fair/unfair). 231 They were asked to complete two ratings (using slider bars initialised at 50) on a scale of 1 to 100 to what extent they believed the responders' decision was motivated by (i) a desire to earn more, and (ii) a desire to reduce the proposer's bonus. These were the measures of self-interest 234 and harmful intent attributions, respectively.

After completing these ratings, proposers were asked to answer a manipulation check, with three possible answers, to measure how cohesive they perceived the responders to be: 1) responders made their decisions as a team, 2) responders made their decisions separately or 3) unsure.

240 We pre-registered 6 main predictions of the study before collecting behavioural data. 
242 P1) Proposers will attribute increased harmful intent to unfair compared to fair responders.

243 P2) Proposers will attribute increased self-interest to unfair compared to fair responders.

244 P3) Proposers will attribute increased harmful intent to responders in cohesive compared 245 to non-cohesive groups.

246 P4) More paranoid proposers will make higher harmful intent attributions overall.

247 P5) More paranoid proposers will show dysregulated responses to opponent cohesion 248 (there will be a paranoia $\mathrm{x}$ cohesion interaction on attributions of harmful intent) 249 P6) Attributions of self-interest will not be related to paranoia.

250

\section{Statistical Approach}

252 We employed an information-theoretic (IT) approach with multi-model averaging (Burnham and 253 Anderson, 2002; Grueber, et al, 2011) to compare the explanatory power of different input 254 variables. The IT approach has many advantages and is widely used in ecology research (see 255 Whittingham et al, 2006 for a detailed review). Unlike null hypothesis significance testing, our 256 method does not employ arbitrary $p$ values as indicators of significance. Rather, we examine the 257 relative support for each model as given by AICc (Akaike Information Criterion, corrected for 258 small sample sizes) values. AIC values estimate the goodness-of-fit of the model to the data 259 (with lower AIC values indicating greater support), while penalising models for the inclusion of 260 additional explanatory terms. Selecting models based on AIC values therefore prioritises 261 parsimony: models should be as simple as possible, but no simpler. This philosophy represents a 262 balance between over-fitting and under-fitting models with too many or too few parameters, 263 respectively. 
265 Our analysis procedure proceeded in four key steps (following Grueber et al, 2011): (i) we

266 specified a full global model containing all terms of interest; (ii) we compared all possible

267 subsets of this model, containing all possible combinations of terms, to one another; (iii) we

268 obtained a 'top model set', containing the subset of models which had equal support (AICc

269 values within 2 units of one another) and (iv) we averaged across this top model set to derive

270 estimates and confidence intervals for the explanatory terms contained in the top model set.

271 Multi-model averaging therefore reduces the reliance on a single 'best' model to obtain

272 parameter estimates and confidence intervals and instead takes into account the fact that there is

273 uncertainty over the true 'best' model.

274

275 We conducted two broad analyses with (i) harmful intent and (ii) self-interest as the respective 276 response terms. We present full (unconditional) model averaged estimates and confidence 277 intervals here, which are more conservative estimates. Due to the extreme skew of the response 278 variables, we converted each one into a 5-level ordered categorical variable (Raihani and Bell, 279 2017). Each term was specified as an ordinal categorical response term in an ordinal logistic 280 regression model, using the $c l m$ function in the ordinal package (Christensen, 2015). In each

281 model, we included the following explanatory terms: paranoia, trust decision (large/small 282 investment), condition (cohesive/non-cohesive), fairness (fair/ unfair), gender and age. Binary 283 input variables were recoded as dummy (1/0) variables.

285 All continuous input variables were standardized (Gelman, 2008) and binary input variables 286 were centered, so estimates can be considered on the same scale. We included incomprehension 
287 (whether subjects answered 1+ comprehension questions incorrectly) in the analyses as a binary 288 dummy variable to control for the effect of failed comprehension on responses. Where 289 comprehension was found to have a meaningful effect, we re-ran analyses including and 290 excluding non-comprehenders to determine whether the models qualitatively changed (no 291 qualitative differences in the results presented below were found).

292

293 Data and code availability

294 The data and code to reproduce the analyses in this study are available in OSF with the identifier 295 https://doi.org/10.17605/OSF.IO/W7YQX. 


\section{Results}

299 The mean $( \pm$ SEM) paranoia score was $50.7 \pm 0.67$, reflecting the combined scores of social 300 reference $(27.1 \pm 0.35)$ and persecution $(23.5 \pm 0.36)$ subscales, respectively. Paranoia scores 301 ranged from 32 to 158 , with 54 participants (4.63\%) being over the clinical mean of 101.9 302 reported in Green et al (2008).

304 As predicted, participants attributed more harmful intent to cohesive compared to non-cohesive 305 opponents (estimate: 0.39, CI: 0.15, 0.64; Figure 1, Table 1) but paranoia did not interact with 306 opponent cohesiveness to exaggerate this effect (estimate: $-0.06, \mathrm{CI}:-0.35,0.24$, Table 1 ). All

307 predictions regarding the validity of the paradigm were supported. Participants attributed more

308 harmful intent to unfair rather than fair opponents (estimate: $-1.19, \mathrm{CI}:-1.43,-0.95$, Table 1) and 309 more self-interest to unfair rather than fair opponents (estimate: -3.37, CI: -3.67, -3.06, Table 2). 310 In addition, paranoia independently and positively predicted harmful intent attribution (estimate:

311 0.77, CI: 0.55, 1.00; Table 1, Figure 2) and there was no effect of paranoia (Figure 2) or 312 opponent cohesiveness on attributions of self-interest (Table 2, Figure 2).

\section{Discussion}

315 We investigated the effects of pre-existing paranoia on harmful intent attribution when 316 interacting with cohesive and non-cohesive groups. Participants played as proposers in an 317 adapted Trust Game against a pair of cohesive or non-cohesive responders. Although the 318 intentions of responders are ambiguous in this task, cohesive responder groups were perceived as 319 more malevolent than non-cohesive responder groups, as measured by harmful intent attributions 
320 made by proposers. Paranoia was associated with a general tendency to make stronger harmful

321 intent attributions in all situations but highly paranoid individuals showed the same increase in

322 harmful intent attribution when interacting with cohesive groups as individuals with lower

323 paranoia. This suggests that pre-existing paranoia reflects a generally heightened tendency for

324 threat perception but an intact sensitivity to group cohesion. Therefore, there was no evidence of

325 a qualitatively nor quantitatively different response to the social context that may reflect a

326 dysregulation in perceiving group interactions.

327

328 This finding converges with studies that found no interaction between paranoia or psychosis and social threat on behavioural and physiological responses (Veling et al, 2016; Counotte et al, 330 2017; Saalfeld et al, 2018). Together, these studies suggest that paranoia is associated with a

331 lower threshold for detecting social threat; but the scaling of response to changes in social threat

332 is similar in all individuals, irrespective of where they lie on the paranoia spectrum. This goes

333 against theories that suggest paranoia involves an increased reactivity to social threat (Yiend et

334 al, 2017; Savulich et al, 2015; Pot-Kolder et al, 2017); and against a generalised insensitivity to 335 social information (Fett et al, 2012, 2016; Gromann et al, 2013).

337 Our results support the hypothesis that group cohesiveness acts as a social threat cue, since 338 paranoid attributions increased in response to greater group cohesiveness. This coincides with 339 evidence that tightly-formed groups are seen as more legitimate subject of conspiracy theories 340 (Grzesiak-Feldman and Suszek, 2008). In light of the Coalitional Index Model (Boyer et al, 341 2015), this would imply that group cohesiveness is an input into the Coalitional Safety Index.

342 Changes in cohesiveness of the responder group affected proposers' attributions of harmful 
343 intent but not self-interest: the responder group was perceived as no more self-interested in

344 conditions of high compared to low cohesiveness, even though cohesive responders were

345 perceived as more malevolent than non-cohesive responders. This can be viewed as a conceptual

346 replication of data showing other social threat cues, group affiliation and social status,

347 independently alter attributions of harmful intent attribution, but not self-interest (Saalfeld et al, 348 2018).

349

350 Despite capturing paranoid ideation scores common to patients with psychosis, it is unclear to

351 what extent these results would characterise patients with paranoid delusions. The mean paranoia

352 score in our sample was similar to that of the non-clinical sample in Green et al.'s original study,

353 with a similar percentage of participants scoring above the clinical mean. However, we did not

354 record whether any participant had a clinical diagnosis and assessing the presence of delusions is

355 best completed with a structured clinical interview, rather than self-report measures. It would be

356 surprising if in such a large sample no participants with delusions were present but we have also

357 noted that severe presentations of delusional paranoia are likely to involve specific changes to

358 social perception that are not present in non-delusional presentations.

359

360 The online nature of the study permitted a large sample size, as well as a more representative

361 sample than typical samples from typical university undergraduate and community participant

362 pools (Berinsky et al, 2012). However, MTurk participants still show important demographic

363 differences to the general population (Huff et al, 2015) and in terms of mental health show

364 higher levels of social anxiety although they are not more likely to report clinically relevant

365 emotional dysregulation than the wider population (Shapiro et al, 2013). Furthermore, given all 
366 our participants were based in the US it is not clear how well these effects might generalise more 367 widely.

368

369 Conclusions

370 Our results replicate and extend existing research in paranoia. Firstly, they suggest that

371 perceptions of opponent group cohesion moderates live paranoid ideation, where more cohesive

372 groups evoke greater perception of threat. Secondly, these results support evidence that those

373 scoring high in paranoia in the general population have a greater tendency to perceive

374 conspiracy, but these attributions of malevolent intent show intact scaling in response to changes

375 in social threat. 
376

377

378

379

380

381

382

383

384

385

386

387

388

389

390

391

392

393

394

395

396

397

398

\section{References}

Bebbington PE, McBride O, Steel C, Kuipers E, Radovanovic M, Brugha T, Jenkins R, Meltzer HI, Freeman D (2013) The structure of paranoia in the general population. Br J Psychiatry 202:419-427. https ://doi.org/10.1192/bjp.bp.112.11903 2

Bentall, R.P., Kaney, S., Bowen-jones, K., (1995). Persecutory Delusions and Recall of Words 1. Cognitive Therapy. Research. 19, 445-457. https://doi.org/10.1007/BF02230411

Berg, J., Dickhaut, J., and McCabe, K. (1995). Trust, reciprocity, and social history. Games and Economic Behavior. https://doi.org/10.1006/game.1995.1027

Berinsky, A.J., Huber, G.A., Lenz, G.S., (2012). Evaluating online labor markets for experimental research: Amazon.com's mechanical turk. Political Analysis. 20, 351-368. https://doi.org/10.1093/pan/mpr057

Boyer, P., Firat, R., van Leeuwen, F., (2015). Safety, threat, and stress in intergroup relations: A coalitional index model. Perspectives on Psychological Science. 10, 434-450. https://doi.org/10.1177/1745691615583133

Burnham K.P., Anderson D.R., (2002). Model Selection and Multimodel Inference: A Practical Information-Theoretic Approach. Springer Verlag: New York

Cameron, N., (1959). The Paranoid Pseudo-Community Revisited. American Journal of Sociology. (Vol. 65). https://doi.org/10.1086/222626

Campbell, D., (1958). Common fate, similarity, and other indices of status of aggregates of persons as social entities. Systems Research and Behavioral Science. 3, 14-25.

Christensen, R. H. B. (2015). ordinal—regression models for ordinal data. R package version, $28,2015$. 
399 Hurvich, C. M., Tsai, C. L., (1989). Time Series Model Selection in Small Samples. Biometrika $400 \quad 76,297-307$.

401 Counotte, J., Pot-Kolder, R., van Roon, A.M., Hoskam, O., van der Gaag, M., Veling, W., 402 (2017). High psychosis liability is associated with altered autonomic balance during 403 exposure to Virtual Reality social stressors. Schizophrenia Research 184, 14-20. $404 \quad$ https://doi.org/10.1016/j.schres.2016.11.025

405 Dasgupta, N., Banaji, M.R., Abelson, R.P., (1999). Group entitativity and group perception: 406 associations between physical features and psychological judgment. Journal of Personality and Social Psychology., 77, 991-1003. doi:10.1037//0022-3514.77.5.991

408 409
Douglas KM, Sutton RM, Cichocka A. (2017). The Psychology of Conspiracy Theories. Current Directions in Psychological Science. 26(6):538-542. doi: 10.1177/0963721417718261

Elahi, A., Perez Algorta, G., Varese, F., McIntyre, J.C., Bentall, R.P., (2017). Do paranoid delusions exist on a continuum with subclinical paranoia? A multi-method taxometric study. Schizophrenia Research. 190, 77-81. https://doi.org/10.1016/j.schres.2017.03.022

Fett, A.K.J., Shergill, S.S., Joyce, D.W., Riedl, A., Strobel, M., Gromann, P.M., Krabbendam, L., (2012). To trust or not to trust: The dynamics of social interaction in psychosis. Brain 135, 976-984. https://doi.org/10.1093/brain/awr359

Fett, A.K.J., Shergill, S.S., Korver-Nieberg, N., Yakub, F., Gromann, P.M., Krabbendam, L., (2016). Learning to trust: Trust and attachment in early psychosis. Psychological Medicine. 46, 1437-1447. https://doi.org/10.1017/S0033291716000015

Freeman, D., (2007). Suspicious minds: The psychology of persecutory delusions. Clinical Psychology Review.. 27, 425-457. https://doi.org/10.1016/j.cpr.2006.10.004 
421 Freeman D, Garety PA, Bebbington PE, Smith B, Rollinson R, Fowler D, Kuipers E, Ray K, 422 Dunn G (2005) Psychological inves- tigation of the structure of paranoia in a non-clinical 423 population. Br J Psychiatry 186:427-435

424 Freeman, D., Garety, P., (2014). Advances in understanding and treating persecutory delusions:

425 A review. Social Psychiatry and Psychiatric Epidemiology. 49, 1179-1189.

426 https://doi.org/10.1007/s00127-014-0928-7

427 Gelman, A., (2008). Scaling regression inputs by dividing by two standard deviations. Statistics $428 \quad$ in Medicine. 27(15), 2865-2873

429 Green, C.E.L., Freeman, D., Kuipers, E., Bebbington, P., Fowler, D., Dunn, G., Garety, P.A., 430 (2008). Measuring ideas of persecution and social reference: The Green et al, Paranoid 431 Thought Scales (GPTS). Psychological Medicine. 38, 101-111.

432 https://doi.org/10.1017/S0033291707001638

433 Gromann, P.M., Heslenfeld, D.J., Fett, A.K., Joyce, D.W., Shergill, S.S., Krabbendam, L., 434 (2013). Trust versus paranoia: Abnormal response to social reward in psychotic illness. 435 Brain 136, 1968-1975. https://doi.org/10.1093/brain/awt076

436 Grueber, C.E., Nakagawa, S., Laws, R.J., Jamieson, I.G., (2011). Multimodel inference in 437 ecology and evolution: Challenges and solutions. Journal of Evolutionary Biology. 24, 699$438 \quad$ 711. https://doi.org/10.1111/j.1420-9101.2010.02210.x

439 Grzesiak-Feldman, M., and Suszek, H. (2008). Conspiracy Stereotyping and Perceptions of Group Entitativity 440 of Jews, Germans, Arabs and Homosexuals by Polish Students. Psychological Reports, 102(3), 755441 758. https://doi.org/10.2466/pr0.102.3.755-758

442 Hamilton, D.L., Sherman, S.J., (1996). Perceiving Persons and Groups. Psychological Review. $443 \quad$ 103, 336-355. https://doi.org/10.1037/0033-295X.103.2.336 
444 Harper, D. J. (1994). Histories of suspicion in a time of conspiracy: a reflection on Aubrey

445 Lewis's history of paranoia. History of Human Sciences, 7(3), 89-109.

446 Hart, A.J., Whalen, P.J., Shin, L.M., McInerney, S.C., Fischer, H., Rauch, S.L., (2000).

447 Differential response in the human amygdala to racial outgroup vs ingroup face stimuli.

$448 \quad$ Neuroreport 11, 2351-2354. https://doi.org/10.1097/00001756-200008030-00004

449 Huff, C., Tingley, D., (2015). "Who are these people?” Evaluating the demographic

450 characteristics and political preferences of MTurk survey respondents. Research and

451 Politics. 2, 205316801560464. https://doi.org/10.1177/2053168015604648

452 Hurvich, C. M., and Tsai, C. L. (1989). Regression and time series model selection in small $453 \quad$ samples. Biometrika, 76(2), 297-307.

454 Jack, A., Egan, V., (2016). Paranoid thinking, cognitive bias and dangerous neighbourhoods:

455 Implications for perception of threat and expectations of victimisation. International

$456 \quad$ Journal of Psychiatry 62, 123-132. https://doi.org/10.1177/0020764015599998

457 Lickel, B., Hamilton, D. L., and Sherman, S. J. (2001). Elements of a lay theory of groups: Types

458 of groups, relational styles, and the perception of group entitativity. Personality and 459 Social Psychology Review, 5(2), 129-140.

460 Lott, A. J., and Lott, B. E. (1961). Group cohesiveness, communication level, and conformity. 461 The Journal of Abnormal and Social Psychology, 62(2), 408.

462 Navarrete, C.D., Olsson, A., Ho, A. K., Mendes, W. B., Thomsen, L., Sidanius, J., (2009). Fear 463 Extinction to an Out-Group Face. Psychological Science. 20, 155-158.

$464 \quad$ https://doi.org/10.1111/j.1467-9280.2009.02273.x 
465 Newheiser, A.K., Sawaoka, T., Dovidio, J.F., (2012). Why do we punish groups? High

466 entitativity promotes moral suspicion. Journal of Experimental Psychology. 48, 931-936.

$467 \quad$ https://doi.org/10.1016/j.jesp.2012.02.013

468 Oyebode, F. (2008). Sims' symptoms in the mind: an introduction to descriptive

$469 \quad$ psychopathology. Elsevier Health Sciences.

470 Pietraszewski, D., Cosmides, L., Tooby, J., (2014). The content of our cooperation, not the color

471 of our skin: An alliance detection system regulates categorization by coalition and race, but 472 not sex. PLoS One 9. https://doi.org/10.1371/journal.pone.0088534

473 Pinkham, A.E., Brensinger, C., Kohler, C., Gur, R.E., Gur, R.C., (2011). Actively paranoid 474 patients with schizophrenia over attribute anger to neutral faces. Schizophrenia Research.

$475 \quad$ 125, 174-178. https://doi.org/10.1016/j.schres.2010.11.006

476 Pot-Kolder, R., Veling, W., Counotte, J., van der Gaag, M., (2017). Self-reported Cognitive

477 Biases Moderate the Associations Between Social Stress and Paranoid Ideation in a Virtual 478 Reality Experimental Study. Schizophrenia Bulletin. 1-8.

479 https://doi.org/10.1093/schbul/sbx119

480 Raihani, N.J., Bell, V., (2017). Conflict and cooperation in paranoia: a large-scale behavioural 481 experiment. Psychological Medicine. 1-11. https://doi.org/10.1017/S0033291717003075

482 Raihani, N.J., Bell, V., (2017). Paranoia and the social representation of others: A large-scale 483 game theory approach. Scientific Reports.. 7, 1-9. https://doi.org/10.1038/s41598-017$484 \quad 04805-3$

485 Raihani, N.J., Mace, R., Lamba, S., (2013). The Effect of \$1, \$5 and \$10 Stakes in an Online 486 Dictator Game. PLoS One 8, 3-8. https://doi.org/10.1371/journal.pone.0073131 
487 Saalfeld V, Ramadan Z, BellV, Raihani NJ. (2018) Experimentally inducedsocial threat $488 \quad$ increases paranoid thinking. Royal Society Open Science.5:

489 180569.http://dx.doi.org/10.1098/rsos. 180569

490

491

492

493

494

495

496

497

498

499

500

501

502

503

504

505

506

507

508

Savulich, G., Freeman, D., Shergill, S., Yiend, J., (2015). Interpretation Biases in Paranoia. Behavioural Therapy. 46, 110-124. https://doi.org/10.1016/j.beth.2014.08.002

Shapiro, D.N., Chandler, J., Mueller, P.A., (2013). Using mechanical turk to study clinical populations. Clinical Psychological Science. 1, 213-220. https://doi.org/10.1177/2167702612469015

Spencer-Rodgers, J., Hamilton, D.L., Sherman, S.J., (2007). The central role of entitativity in stereotypes of social categories and task groups. Journal of Personality and Social Psychology. 92, 369-388. https://doi.org/10.1037/0022-3514.92.3.369

Taylor, M.J., Freeman, D., Ronald, A., (2016). Dimensional psychotic experiences in adolescence: Evidence from a taxometric study of a community-based sample. Psychiatry Research. 241, 35-42. https://doi.org/10.1016/j.psychres.2016.04.021

Veling, W., Pot-Kolder, R., Counotte, J., Van Os, J., Van Der Gaag, M., (2016). Environmental Social Stress, Paranoia and Psychosis Liability: A Virtual Reality Study. Schizophrenia Bulletin. 42, 1363-1371. https://doi.org/10.1093/schbul/sbw031

Whittingham, M.J., Stephens, P.A., Bradbury, R.B., Freckleton, R.P., (2006). Why do we still use stepwise modelling in ecology and behaviour? J. Anim. Ecol. 75, 1182-1189. https://doi.org/10.1111/j.1365-2656.2006.01141.x

Yiend, J., Trotta, A., Meek, C., Dzafic, I., Baldus, N., Crane, B., Kabir, T., Stahl, D., Heslin, M., Shergill, S., McGuire, P., Peters, E., (2017). Cognitive Bias Modification for paranoia 
509 (CBM-pa): Study protocol for a randomised controlled trial. Trials 18, 1-13.

$510 \quad$ https://doi.org/10.1186/s13063-017-2037-x 


\section{Table $\mathbf{1}$ (on next page)}

Information for the ordered logistic regression investigating the attribution of selfinterest to responder pairs in the trust game.

Model averaged estimates, unconditional standard errors, confidence intervals and relative importance for the terms included in the top model set for the ordered logistic regression investigating the attribution of self-interest to responder pairs in the trust game. See supplementary material for top model set. Reference levels are shown in parentheses. 


\begin{tabular}{|c|c|c|c|c|}
\hline Parameter & Estimate & $\begin{array}{l}\text { Unconditional } \\
\text { SE }\end{array}$ & $\begin{array}{l}\text { Confidence } \\
\text { Interval }\end{array}$ & $\begin{array}{l}\text { Relative } \\
\text { Importance }\end{array}$ \\
\hline Intercept $1 \mid 2$ & -2.84 & 0.11 & $(-3.06,-2.63)$ & \\
\hline Intercept $2 \mid 3$ & -1.97 & 0.09 & $(-2.15,-1.78)$ & \\
\hline Intercept $3 \mid 4$ & -1.26 & 0.09 & $(-1.43,-1.09)$ & \\
\hline Intercept $4 \mid 5$ & 0.05 & 0.08 & $(-0.10,0.21)$ & \\
\hline Fairness (1=fair) & -3.37 & 0.15 & $(-3.67,-3.06)$ & 1.00 \\
\hline Gender ( $1=$ male $)$ & -0.07 & 0.12 & $(-0.31,0.16)$ & 0.43 \\
\hline Comprehension $(1=>$ & -0.09 & 0.19 & $(-0.46,0.29)$ & 0.34 \\
\hline $\begin{array}{l}1 \text { comprehension } \\
\text { failure) }\end{array}$ & & & & \\
\hline $\begin{array}{l}\text { Trust Decision } \\
\text { (1=sent higher } \\
\text { amount) }\end{array}$ & 0.06 & 0.12 & $(-0.18,0.29)$ & 0.35 \\
\hline Cohesion & 0.002 & 0.03 & $(-0.06,0.06)$ & 0.06 \\
\hline Paranoia & -0.0021 & 0.03 & $(-0.07,0.06)$ & 0.06 \\
\hline
\end{tabular}


Figure $\mathbf{1}$ (on next page)

Box plot to show harmful intent attribution made by proposers concerning pairs of responders

Each data point indicates the harmful intent each proposer attributed to the responder team they interacted with, according to the cohesiveness of responders, and whether responders' decisions were fair (a) or unfair (b). 


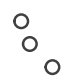

$75-$

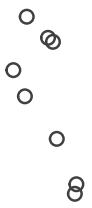

8

o

$50-$

$25-$

$0-$

$\circ$ $\circ$

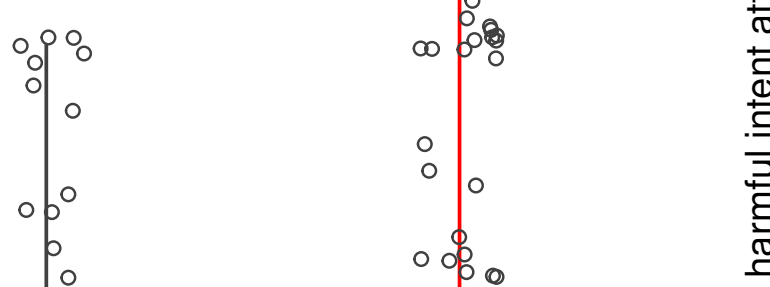

Peer] reviewing PDF | (2019:04:36566:1:1!NEW 24 Jun 2019) non cohesive non co'hesive
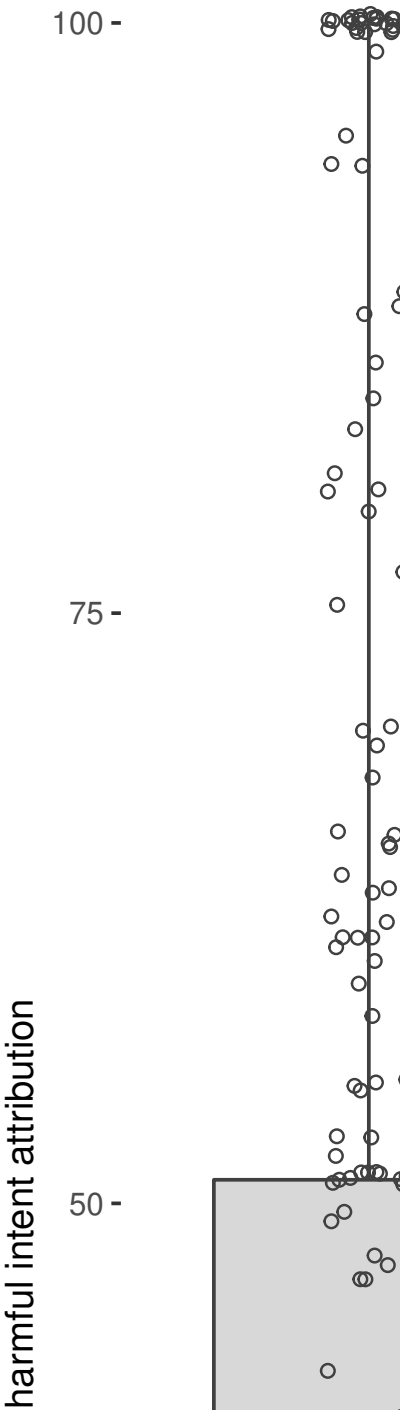

0

○

$\circ$

$\circ$

sop

$\circ$

$50-$

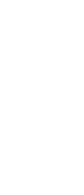

$25-$

$0-$

$8 \circ$

-

$8 \infty^{\circ}$

$\circ$

O 0

(1) 0 कos

8े

$\circ 0$

$\circ 0_{0}^{0}$

$\circ$

$\infty$

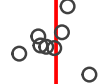

$\circ \%$

$\circ 0$

$\circ$

م

o

8

008

০০

0
0
0
000
0
0

$\infty$

- 0

- 8

$\infty$

-

80

.

$0_{0}^{\infty} 0$

$\circ 8$

800

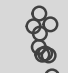

유이

- 0 \%०

80

80 O)

880
कि०

○ం

- 82

- 8

वृ8 0

200

०0 8

800

80

8

-

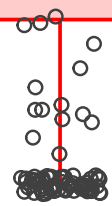

cohesive 


\section{Table 2 (on next page)}

Information for the ordered logistic regression investigating the attribution of harmful intent to responder pairs in the trust game

Model average estimates, unconditional standard errors, confidence intervals and relative importance for the terms included in the top model set for the ordered logistic regression investigating the attribution of harmful intent to responder pairs in the trust game. See supplementary material for top model set. Reference levels are shown in parentheses. 


\begin{tabular}{|c|c|c|c|c|}
\hline \multirow[t]{2}{*}{ Parameter } & \multirow{2}{*}{ Estimate } & \multirow{2}{*}{$\begin{array}{l}\text { Unconditional } \\
\text { SE }\end{array}$} & \multirow{2}{*}{$\begin{array}{l}\text { Confidence } \\
\text { Interval }\end{array}$} & \multirow{2}{*}{$\begin{array}{l}\text { Relative } \\
\text { Importance }\end{array}$} \\
\hline & & & & \\
\hline Intercept $1 \mid 2$ & 0.42 & 0.07 & $(0.29,0.54)$ & \\
\hline Intercept $2 \mid 3$ & 1.31 & 0.08 & $(1.17,1.46)$ & \\
\hline Intercept $3 \mid 4$ & 2.04 & 0.09 & $(1.86,2.22)$ & \\
\hline Intercept $4 \mid 5$ & 2.76 & 0.12 & $(2.53,2.99)$ & \\
\hline Cohesion $(1=$ cohesive $)$ & 0.39 & 0.12 & $(0.15,0.63)$ & 1.00 \\
\hline Fairness (1=fair) & -1.19 & 0.12 & $(-1.44,-0.95)$ & 1.00 \\
\hline Gender $(1=$ male $)$ & -0.36 & 0.13 & $(-0.60,-0.11)$ & 1.00 \\
\hline Comprehension $(1=>1$ & 0.86 & 0.22 & $(0.43,1.29)$ & 1.00 \\
\hline \multicolumn{5}{|l|}{ comprehension failure) } \\
\hline Trust decision $(1=$ sent & -0.34 & 0.14 & $(-0.62,-0.05)$ & 1.00 \\
\hline \multicolumn{5}{|l|}{ larger amount) } \\
\hline Paranoia & 0.77 & 0.11 & $(0.55,1.00)$ & 1.00 \\
\hline Cohesion:Paranoia & -0.06 & 0.15 & $(-0.35,0.24)$ & 0.32 \\
\hline
\end{tabular}

1 
Figure 2 (on next page)

Mean harmful intent and self-interest attributions made by participants

Data points indicate the harmful intent (black) and self-interest (red) attributions made by participants in (a) the cohesive responder condition and (b) the non-cohesive responder condition. Means and standard errors are generated from raw data. Paranoia was converted to a five-level categorical variable for ease of visualisation, although it was included as a continuous term in the models. 

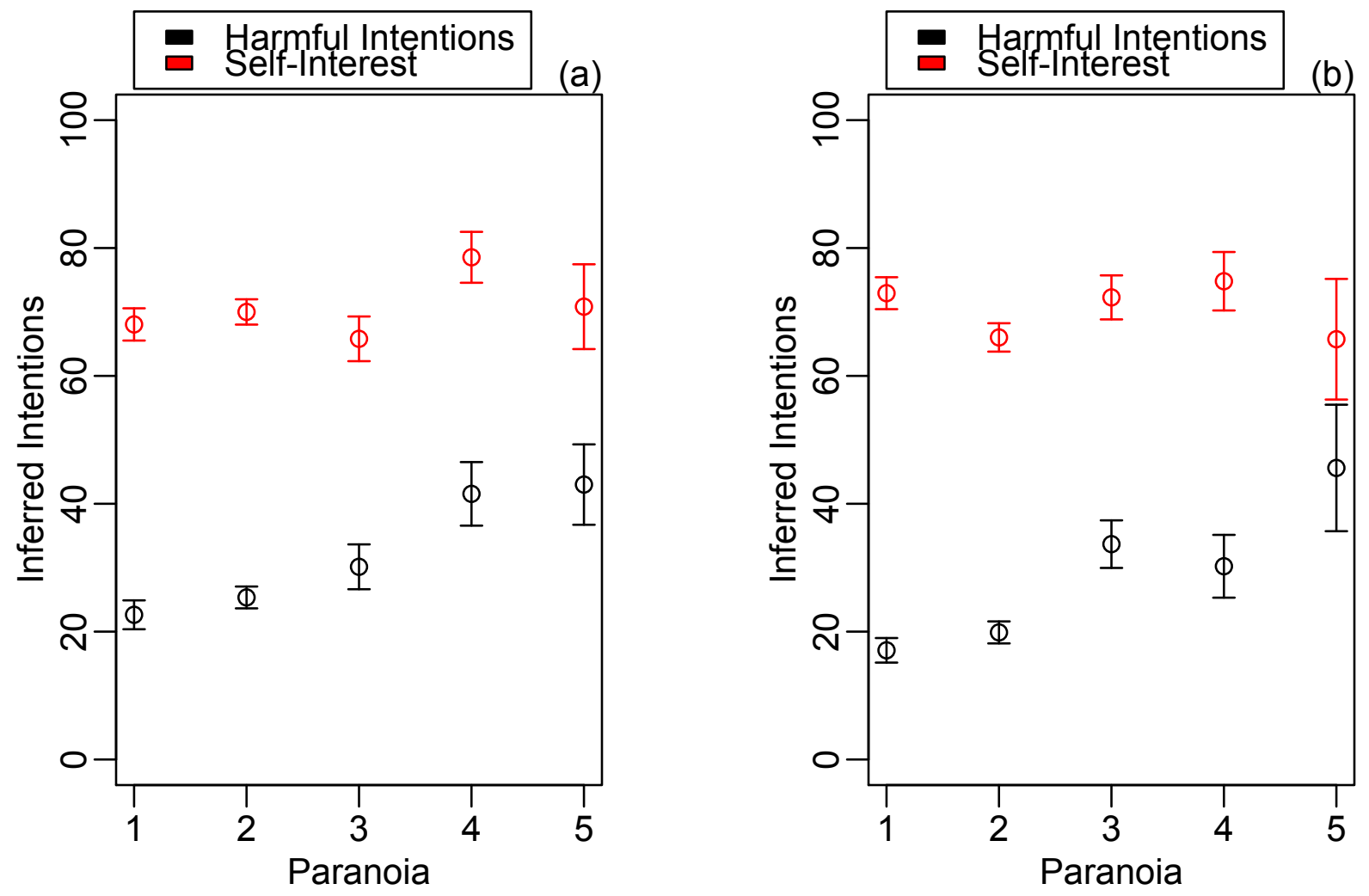